\title{
Studies on stress-induced modulation of long term potentiation in rodent hippocampus: what can we learn about pathogenesis of depression?
}

\author{
Natalia V.Gulyaeva* \\ Institute of Higher Nervous Activity and Neurophysiology RAS, Russia
}

\begin{abstract}
Long-term potentiation (LTP) reflecting continuous changes in synaptic efficacy is regarded as a cellular model of learning and memory. In this mini-review the data relating to hippocampal LTP alterations in rodent models of depression are analyzed to learn whether disturbances in LTP may be reliable markers of synaptic plasticity impairments underlying depressive and anxiety states. LTP disturbances result from synaptic reorganizations induced by multiple inter-related and mutually dependent events: hypothalamic-pituitary-adrenal axis disfunction; malfunction of neurotransmitter systems; failure to maintain the balance of neurotrophic systems; neuroinflammatory processes; disturbance in neurogenesis. Stable deficits in hippocampal LTP reflect the synapse-related basic mechanisms for cognitive and emotional behavioral deficits characterisic for depression/anxiety, and altered LTP is indicative of the development of stress-induced psychopathology.
\end{abstract}

\begin{abstract}
Abbreviations: BDNF: Brain-Derived Neurotrophic Factor; BNST: Bed Nucleus of the Stria Terminalis; CB1: Cannabinoid Receptor1; CFC: Contextual Fear Conditioning; CMS: Chronic Mild Stress; CS: Corticosterone; CUS: Chronic Unpredictable Stress; DG: Dentate Gyrus; GluR: Glutamate Receptor; GRs: Glucocorticoid Receptors; HPA: Hypothalamic Pituitary Adrenal; LTD: Long-Term Depression; LTP: Long-Term Potentiation; MS: Maternal Separation; SI: Social Isolation Stress; UWT: Underwater Trauma
\end{abstract}

\section{Literature}

Chronic stress is considered to be a major risk factor in the development of mood diseases. In order to study mechanisms involved in the etiology of human affective disorders, there is an abundant use of various animal models based on different stress paradigms. Depressive disorders are the most common psychiatric pathology, and a great number of people who experience depression also experience anxiety. Sometimes, anxiety is more expressed than depression, while in many cases neither is clearly predominant. In rodent stress-based models anxiety and depression symptoms often are demonstrated in parallel.

Activity-dependent changes in synaptic strength are widely accepted as key mechanisms for information storage in the brain [1]. Long-term potentiation (LTP) is an electrophysiological phenomenon, an hours-lasting increase of postsynaptic potentials after tetanization. It is believed to reflect long-term changes in synaptic efficacy in distributed networks, associated with constant changes in the behavioral phenomena, which are often interpreted as the retention of information [2]. Thus, hippocampal LTP is regarded as a cellular model of learning and memory. Cognitive impairments and LTP impairments are increasingly recognized as events accompanying and reflecting experimental depression, anxiety and other experimental stress-related chronic psychological disorders, therefore, disturbances in LTP may be reliable markers of synaptic plasticity impairments underlying depressive and anxiety states. The dentate gyrus (DG) of the hippocampus is supposed to play a critical role in defining the impact of stress on hippocampal functioning. The DG is a dynamic structure susceptible to stress, its variable and complex stress response being reflected in changes of LTP and local circuit activity associated with the behavioral outcomes [3].

Structural and neurochemical changes in the hippocampus have been found in major depressive disorder, these changes being pivotal for regulation of mood and cognitive functions. Stressful life events, specifically inducing fear, provoke adaptive anxiety that can also lead to exaggerated states, forming a link of mental illnesses pathogenesis. These states are associated with alterations in dendritic and synaptic structure within specific brain regions, including the hippocampus, amygdala, and prefrontal cortex (PFC) [4]. Stress-induced structural plasticity underlies changes in hippocampal plasticity recorded using electrophysiological approaches. Exposure to experimental stress induces atrophy and cell loss in the hippocampus accompanied by decreased expression of neurotrophic/growth factors, while antidepressants reverse the effects of stress upregulating neurotrophic factor systems and neurogenesis in the adult hippocampus [5-7]. The key role of adult neurogenesis and its abnormalities in the development of depression have become widely debated during the last decade, the main bulk of the data that form the basis of this hypothesis having been obtained from rodent model experiments [8]. Hippocampal LTP is critically dependent on neurogenesis in the subgranular

Correspondence to: Natalia V.Gulyaeva, Institute of Higher Nervous Activity and Neurophysiology RAS, 5a Butlerov Street, Moscow, 117485, Russia, E-mail: nata_gul@pisem.net

Key words: depression, anxiety, synaptic plasticity, long term potentiation (LTP), stress

Received: April 14, 2016; Accepted: August 05, 2016; Published: August 09, 2016 
neurogenic niche. Linking depressive disorders to adult hippocampal neurogenesis, Schinder \& Gage [9] have proposed a hypothesis about the contribution of specific features of newly generated neurons to hippocampal plasticity; later Perera et al. [10] speculated that stressinduced suppression of neurogenesis would uncouple emotions from external context leading to a negative mood state, its persistence inducing depression. Neuroinflammatory processes negatively affect both neurogenesis and synaptic plasticity $[11,12]$. For example, old autoimmune-prone cytokine B-cell-activating factor (BAFF) transgenic mice exhibit an anxiety-like phenotype associated with brain inflammation and decreased proliferation of newly formed neurons in the subgranular zone of the hippocampus; they also demonstrate an abnormal neuronal activation within the limbic system in response to mild anxiogenic stimuli and impaired neurogenesisdependent and neurogenesis-independent LTP in DG [13]. These data support the idea that neuroinflammation, including inflammatory processes accompanying autoimmune diseases, may be associated with emotional disorders.

Tanti \& Belzung [14] have analyzed relations of rodent depression models and of antidepressant therapies to adult neurogenesis along the septo-temporal axis of the hippocampus The dorsal hippocampus (septal part) is responsible for cognitive function (learning, memory and spatial navigation), while the ventral hippocampus (temporal part) is involved in the control of emotional and anxious behaviors and regulation of the neuroendocrine stress axis. Indeed, stressresponse is different in the dorsal and ventral parts of hippocampus. Studies on rodent models of stress and depression in search of links between stress, psychiatric diseases and changes in the hippocampus (corticosteroid receptors, neurotransmitter systems, neurotrophins, neurogenesis, plasticity/LTP) strongly suggest a specific involvement of ventral hippocampus in the development of depression $[15,16]$. Expectedly, effects of stress on the neurogenic niche are restricted to the ventral hippocampus more frequently than they are evident in the dorsal part. In addition, the effects are also stage specific, and concern mostly neurogenesis rather than cell proliferation or survival [14]. Surprisingly, antidepressants: selective serotonin re-uptake inhibitors as well as non-pharmacological manipulations that are also endowed with antidepressant effects, such as environmental enrichment or physical exercise, act in a more uniform way on dorsal and ventral adult neurogenesis. Dine et al. [17] revealed a multifaceted highanxiety neurophysiological endophenotype in the murine ventral hippocampus by applying electrophysiological techniques to brain slices from animals of the high anxiety-related behavior (HAB) and normal anxiety-related behavior (NAB) mouse model. Interestingly, basal neurotransmission at ventral hippocampal CA3-CA1 synapses was weaker in $\mathrm{HAB}$ as compared to NAB mice, while paired-pulse facilitation and LTP at these synapses were more pronounced in slices from $\mathrm{HAB}$ animals. Intranasal administration of neuropeptide $S$, a potential novel treatment option for anxiety, increased basal neurotransmission and reduced paired-pulse facilitation and LTP in slices from $\mathrm{HAB}$ animals shifting this endophenotype towards norm.

Stress exerts its effect on cognitive function and mood to the utmost through the actions on hypothalamo-pituitary-adrenal (HPA) axis, in particular via effects of stress hormone corticosterone (CS) on synaptic plasticity. Increased levels of CS affect hippocampal functioning via the glucocorticoid receptors (GRs). A number of studies reported stressinduced alterations in hippocampal LTP depending on the nature and duration of stress [18], however only few of these studies are directly linked to CS effects and confirm that stress paradigms used model depressive behaviors. Kamal et al. [19] showed that susceptibility to stress following social isolation stress (SI), in particular genetic background, has a major effect on the level of in vitro LTP impairment in the CA1 hippocampal area in mice. SI significantly increased CS levels. Chronic CS infusion in control mice phenocopied the LTP impairments observed in SI mice, while infusion of the GRs antagonist RU38486 rescued the LTP impairments induced by SI. Exposure of young rats to tail suspension stress for $30 \mathrm{~s}$ significantly increased serum CS and attenuated CA1 LTP in hippocampal slices [20]. This effect was mediated by CS-dependent increase in postsynaptic $\mathrm{Zn}^{2+}$ signal. The data suggest that changes in hippocampal function induced by acute stress may be a potential risk factor under chronic stress circumstances to induce depressive symptoms.

Along with disturbances in the HPA axis, the most common stressrelated psychiatric disorders (depression and generalized anxiety) are underlain by dysfunctions of neurotransmitters systems, in particular serotoninergic (5-HT) and glutamatergic. Indeed, disturbances in 5-HT/HPA interactions at molecular and functional levels and related alterations of hippocampal neurogenesis are key links in the pathogenesis of psychoaffective disorders [21]. Studies on LTP in the hippocampal CA1 field in 5-HT-depleted rats suggest that the serotonergic mechanism is involved in the psychological stress-induced alteration in synaptic plasticity [22]. Epigenetic modulation of specific genes related to development of pro-depressive or antidepressive stress response (5HT transporter and receptors, corticotropin releasing hormone, glucocorticoid and their receptors, BDNF and other neurotrophic factors) may contribute to the formation of depressive endophenotypes [23].

Besides the monoamine system that has been the central focus of neurobiological research into depression and represents the major target of modern antidepressant drugs, dysfunction of the glutamatergic system has also emerged as a major pathological feature in depression. A most important excitatory neurotransmitter, glutamate, is a key player in synaptic plasticity phenomena, in particular LTP and new synapse formation. The experiments performed in cats and rats by Adamec et al. [24] confirmed that NMDA-dependent LTP of limbic system circuits controlling defensive behavior underlies stressor-induced permanent increases in anxiety-like behavior. Dysfunction of glutamate receptors (a-amino-3-hydroxy-5-methylisoxazole-4-propionic acid subtype of glutamate receptors, AMPAR; N-methyl-D-aspartate receptors, NMDAR and metabotropic glutamate receptors, mGluR) may account for alterations to multiple signal transduction pathways in depression [25]. Concepts supporting a NMDAR hypothesis of depression postulate that the pathogenesis of depression may arise from stressors inducing excessive NMDAR activity which acts at both cellular and regional levels stimulating the depressive phenotype. Such NMDAR dysfunction may lead to alterations of synaptic efficiency/LTP pathways in hippocampus and other regions (e.g. amygdala). Glutamatergic processes are essentially implicated in the pathophysiology and treatment of depression, including the antidepressant effects of NMDAR receptor antagonists. Kudryashova [26] has reviewed the data on the involvement of impairments of glutamatergic system functions in the development of depressive states in humans and animals and discussed the hypothesis on the contribution of excitotoxic damage to neurons in depression. An alternative hypothesis was also considered dealing with deadaptation of the mechanisms of presynaptic, including structural, plasticity due to a deficit of trophic factors. Indeed, the interrelations between glutamatergic system and neurotrophins may unify these hypotheses into one concept. CS released during stress 
response is able to alter functional properties of synaptic glutamate receptors, in particular AMPAR and NMDAR responsible for the expression of synaptic plasticity. Stress levels of CS applied to adult rat hippocampal slices potentiated evoked NMDAR-mediated synaptic responses as well as facilitation of both LTP and long term depression (LTD) [27]. A CS-mediated slow-onset increase in GluN2A receptors in hippocampal synapses revealed in this study could be a homeostatic mechanism to normalize synaptic plasticity following fast-onset stressinduced facilitation.

Repeated stress is one of the environmental factors that precipitates and exacerbates depression and anxiety as well as cognitive impairments. A variety of approaches, first of all different types of stress applied to different rodents of different gender and age, make it difficult to compare the results from various groups. However, considering both sides of the coin, the diversity of existing models is quite positive since it allows performing targeted studies of selected neurobiological paradigms/mechanisms of depressive state development, as well as to investigate mechanisms of action and predict pharmacological profiles of potential antidepressants $[28,29]$.

As compared to men, women have increased stress susceptibility and exhibit a nearly twofold increased risk of developing depression and anxiety disorders. Chronic stress, sex and 5-HT deficiency are recognized main risk factors in the development of aberrant emotional behavior typical for psychiatric diseases. Indeed, effects of stress on LTP are gender-specific. A study using a contextual fear conditioning (CFC) paradigm demonstrated that the females exhibited low anxiety-like behavior, and CFC increased extracellular 5-HT levels in the hippocampus only in the males. LTP in hippocampal CA1 was suppressed by CFC in the males only, and pretreatment with the 5-HT1A receptor agonists tandospirone suppressed LTP induction in the males, while synaptic responses to CFC and 5-HT1A receptor interventions were not observed in the females [30].

Chronic unpredictable stress (CUS) or chronic mild stress (CMS) paradigms are among most popular and relevant models of stressinduced depression/anxiety behaviors in rodents. As a rule, in these models rather mild stimuli are used more frequently than painful ones to induce depressive behaviors and anxiety. CUS in rats induced depressive-like behavior (diminished sucrose preference and reduced measures of locomotor activity), impaired LTP in the hippocampal CA1 region and significantly decreased synaptophysin mean density in the hippocampal CA3 region and respective mRNA levels [31]. Electroconvulsive stimulation improved CUS-induced behavioral effects, synaptophysin deficits and ameliorated LTP impairment. Changes in hippocampal synaptic plasticity were dependent on the duration of CUS, the functional changes at CA3-CA1 hippocampal synapses occurring earlier than the structural alterations. Qiao et al. [32] reported that CUS during three weeks induced depressive-like behaviors, impaired LTP induction, and decreased basal synaptic transmission at hippocampal CA3-CA1 synapses recorded in vivo; a decrease in density of dendritic spines in CA1 and CA3 pyramidal neurons and brain-derived neurotrophic factor (BDNF) level in the hippocampus accompanied changes in synaptic plasticity. After a two week CUS, some depressive-like behaviors were observed, accompanied by depressed basal synaptic transmission, enhanced LTP induction at the CA3-CA1 synapses and decreased BDNF. After a 7 day long CUS, most indices did not differ from control yet. CUS induced depressive-like behaviors and decreased the number of bromodeoxyuridine-labeled neural progenitor cells and doublecortinpositive immature neurons in the DG of mice [33]. Simultaneously,
CUS impaired induction of neurogenesis-dependent LTP in the DG. Close relationship between behavioral, cellular and LTP deficits in CUS-exposed mice was further confirmed by prevention all of them by a chronic treatment with a monoacylglycerol lipase inhibitor JZL184.

Hippocampal microglial activation may play a causal role in chronic stress-induced cognitive and LTP disturbances. Liu et al. [34] demonstrated that a rat model of CUS induced a cognitive impairment in spatial memory and the Morris water maze test and a hippocampal LTP impairment accompanied by microglial activation and attenuated phosphorylation of glutamate receptor 1 (GluR1 or GluA1). Blockade of microglial activation by chronic treatment with minocycline before CUS prevented CUS-induced impairments of both spatial memory and LTP induction as well as increased phosphorylation of GluR1.

CMS significantly modified physiological and behavioral reactions, as observed by the impairment in avoidance extinction and LTP in the hippocampal-accumbens pathway, and the alterations in depressionlike symptoms, such as coping with stress behavior, weight gain, and sucrose consumption [35]. Exposure to CMS produces downregulation of the cannabinoid 1 (CB1) receptor in the hippocampus of male rats. Enhancing cannabinoid signaling using the CB1/2 receptor agonist WIN55,212-2 and GRs antagonist RU-38486 prevented the CMSinduced alterations in extinction and plasticity. The involvement of endocannabinoids in the stress response and their putative contribution to the etiology of mood disorders were confirmed by Reich et al. [36] , who reported that CMS (21 d) in rodents significantly altered hippocampal endocannabinoid-mediated neurotransmission and synaptic plasticity in in vitro experiments (field potential recordings, LTP in hippocampal area CA1). The results suggest that CMS does not directly affect glutamatergic neurotransmission, rather CMS sensitizes $\mathrm{CB} 1$ function on GABAergic terminals, leading to less inhibition and an increase in excitatory neurotransmission. Interestingly, LTP-blocking property of WIN 55,212-5 shifted from being glutamate-dependent in non-stress animals to being GABA-dependent in CMS animals.

In addition to CUS/CMS, different paradigms of early life stress (including acute stress) are very popular models of depression/anxiety in adult rodents. Traumatic events during early life may affect the neural systems associated with memory function, including extinction, and, most importantly, lead to altered sensitivity to stress later in life. Exposure to chronic stress during the neonatal period is known to induce permanent long-term changes in the central nervous system and HPA axis reactivity that are associated with increased levels of depression, anxiety, and cognitive impairments. Neonatal proinflammatory stress (bacterial lipopolysaccharide or interleukin administration) induces "perinatal malprogramming" resulting in development of depressionlike behaviors, associated with abnormalities in functioning of the HPA axis, impaired hippocampal neuroplasticity (LTP) and changes in hippocampus-dependent memory formation [37]. As compared to neocortex, hippocampus was shown to be more vulnerable to stressinduced inflammation and demonstrated persistent inflammatory response induced by chronic stress. This maladaptive inflammatory response was associated with a selective increase in hippocampal CS accumulation and changes in CS signaling [38].

Maternal separation (MS) paradigm in rodents is a validated model of early life stress associated with increased depression, anxiety, and cognitive impairments in adults. The neuronal and endocrine changes induced by MS are long lasting, and exaggerate the mild ageassociated deficits. Sousa et al. [39] demonstrated that the baseline behavioral deficits of aged (70-week-old) control rats were further 
aggravated by MS, indicating that early-life stress-induced continuous changes in anxiety-like behavior and hippocampal-dependent memory were maintained later in life. These differences were associated with impaired hippocampal plasticity: the magnitude of hippocampal LTP from Schaffer collaterals/CA1 synapses was significantly lower in aged MS animals than in age-matched controls. Early deprivation model which involves both dam and littermate deprivation is less investigated in comparison with classical MS. Zhang et al. [40] reported that this paradigm of early deprivation reduced threshold of LTP induction in hippocampal CA3-CA1 pathway, lowered CS, reduced anxiety and enhanced memory (better performance in social recognition and Morris water maze test). The authors suggested that the surprisingly diminished HPA axis response and facilitated hippocampal LTP may contribute to the anxiety-reducing and memory-enhancing effects of early deprivation, respectively.

An increased incidence of anxiety, depression and attention deficits in children is believed to be linked to psychological stress during pregnancy. Stress to a pregnant rat during the period when the foetal limbic and HPA axes develop induces anxiogenic and depressive behaviours as well as learning and attention deficits in the offspring. These consequences of prenatal/maternal stress depend on its intensity and timing as well as on offspring gender. For example, maternal stress increased CS levels in the foetal brain, reduced foetal testosterone and brain aromatase activity in males, while it altered brain catecholamine activity in females [41]. Learning deficits, reductions in hippocampal neurogenesis, LTP and dendritic spine density in the prefrontal cortex were more expressed in prenatally-stressed males, while anxiety, depression and increased response of the HPA axis to stress were more prevalent in females.

The sound stress during fetal life induced anxiety, efficiently disturbed both cognitive abilities and synaptic plasticity in male Wistar rats. It negatively affected the basic synaptic responses and led to a lower level of LTP assessed in the CA3-CA1 pathway of the hippocampus [42]. The offspring of pregnant females exposed to the noise stress had an elevated level of CS suggesting that changes in HPA axis might contribute to LTP disturbances. Using a chronic prenatal restraint stress model where the rat dams were immobilized for $45 \mathrm{~min}$ three times per day during the last week of pregnancy Yeh et al. [43] found that stress switched the direction of synaptic plasticity in hippocampal CA1 region, favouring low-frequency stimulation-induced LTD and depressing the induction of LTP by high-frequency stimulation in young (5-week-old) rat offspring, these changes disappearing at adulthood (8 weeks old). Interestingly, fostering of prenatal restraint stress offsprings to control dams did not alter the effects of stress on LTP and LTD. Prenatal stress-induced changes in LTP and LTD induction correlated with increases in non-cleaved pro-BDNF and decreases in the mature BDNF (mBDNF) levels as well as with decrease in the expression of tissue plasminogen activator, a protease involved in the extracellular conversion of pro-BDNF to mBDNF. In these experiments, no gender differences could be revealed.

Since the majority of stress stimuli in humans leading to psychopathology are of social nature, studies of consequences of social stress in adult animal models are very relevant. One of these models uses the resident-intruder paradigm, in particular social defeat in rats [44]. A differential effect of a brief double social defeat and repetitive social defeat stress on dendritic remodeling in hippocampal CA3 neurons has been described, this effect being associated with alterations of hippocampal LTP and LTD. Collins [45] suggested a conflict model, developed to generate a more naturalistic model of anxiety, utilizing two non-noxious stressors (predator (cat) odor and light), external stimuli eliciting typical, stimulus-specific, anxiety-related behaviors. Ex vivo induction of LTP within the CA1 region of the hippocampus was reduced following exposure to light stress, independent of presence, or absence, of odor. However, after a single presentation, LTP was diminished following either odor presentation or dual presentation of the stressors. LTP responses in hippocampus obtained from conflictexposed animals were hemisphere-specific. Ardi et al. [46] examined the impact of an underwater trauma (UWT) reminder on anxietylike behaviour, and electrophysiological indices in the hippocampus and the amygdala. The exposure to UWT by itself increased anxiety behaviour and paired-pulse inhibition in the DG. UWT reminder $24 \mathrm{~h}$ later resulted in an additional increase in anxiety behaviors and pairedpulse inhibition accompanied by impaired LTP in DG.

Data on the depression/anxiety-related effects of stress conditions on LTP in brain structures other than hippocampus are quite limited. CUS induced anhedonia associated with lower amplitude of field excitatory postsynaptic potential in the PFC [47]. Chronic restraint stress provoked receptor-mediated impairments to catecholaminergic facilitation of synaptic plasticity in the medial PFC (intra-infralimbic LTP) [48]. LTP-like response in the hippocampal-medial PFC pathway was associated with extinction retrieval of context-dependent fear memory. Early postnatal stress induced neurodevelopmental dysfunction of this neural circuit and impaired fear extinction later in life [49]. Chronic restraint stress facilitated synaptic plasticity in the anterior cingulate cortex (field excitatory postsynaptic potentials duration, paired-pulse ratios and LTP level) via increased excitability due to disinhibition of GABA(A) receptor signalling, which may underlie induction of stress-related behavioral hyper-locomotive activity [50]. Adamek [51] showed that LTP in periacqueducatal gray mediated stress induced increases in anxiety in rodents, as it did in the cat. Cocaine withdrawal enhanced NMDA receptorsdependent LTP induced by corticotropin-releasing factor at central amygdala glutamatergic synapses [52]. In the lateral amygdala LTP was dependent on the metabotropic glutamate receptor subtype 7 (mGlu7), an important presynaptic regulator of neurotransmission linked to anxiety and depression [53] and was gated by the striatal-enriched protein tyrosine phosphatase [54]. Thirty days after the last exposure to chronic restraint stress for 2 weeks late adolescent rats demonstrated increased anxiety, impaired LTP in the ventral subiculum-nucleus accumbens (NAc) pathway, impaired performance in the PFCdependent object-recognition task and the hippocampal-dependent spatial version of this task, and significantly reduced expression of GRs in the amygdala, hippocampus, PFC, and NAc[ 55]. The bed nucleus of the stria terminalis (BNST), a subregion of the extended amygdala, is regarded as a relay of corticolimbic information to the paraventricular nucleus of the hypothalamus to directly influence the stress response. Conrad et al. [56] showed that chronic CS injections and chronic SI housing conditions induced anxiety-like behavior in mice, and chronically stressed mice also displayed a parallel blunting of LTP in the dorsal-lateral BNST. Conversely, acute SI housing had no effect on anxiety-like behavior but still resulted in a blunting of LTP in the BNST. Thus, acute and chronic stressors specifically affect synaptic plasticity in the BNST, these changes being not consistently associated with an increase in anxiety-like behavior.

Plasticity is a specific feature of the nervous system underlying key aspects of learning, memory, and repair. Dysregulation of neuroplasticity, in particular synaptic plasticity, contributes to numerous neuropsychiatric diseases. Discussing possible mechanisms 
of ambiguous stress-reactivity of long-term plasticity [18], it has been concluded that vast unpredictability of stress induced modulation of synaptic plasticity has various reasons. In acute stress conditions, stress paradigms, severity, duration, and period of recording, brain structure and other factors enhancing uncertainty and unpredictability of stress response are important, their action is highly regulated by emotional experience, and changes of synaptic plasticity may be transient. Stress paradigms modeling depression/anxiety are dealing with chronic states, as are depressive and anxiety disorders in human. In this situation changes of synaptic plasticity are much more predictable and are accompanied by definite behavioral and molecular alterations. Indeed, behavioral manifestations of these pathologies reflect altered level of synaptic plasticity. In most clinically relevant models of depression/ anxiety hippocampal LTP is a mirror of disturbed plasticity. LTP disturbances result from synaptic reorganizations induced by multiple inter-related and mutually dependent events, primarily: HPA axis disfunction affecting CS and its receptors in the brain; malfunction of neurotransmitter systems; failure to maintain the balance of neurotrophic factors and their receptors; neuroinflammatory processes; disturbance in neurogenesis (including proliferation, differentiation, and migration) modifying the appearance of new neurons. On the one hand, stable deficits in hippocampal LTP (as well as of LTP in other structures of the limbic system) reflect the synapserelated basic mechanisms for cognitive and emotional behavioral deficits characterisic for depression. On the other hand, altered LTP is indicative (or even predictive) of the development of stress-induced psychopathology, representing its distinctive biomarker useful for deeper studies of synaptic pathogenetic mechanisms of mental diseases.

\section{Acknowledgement}

This study was supported by the Russian Science Foundation (RSF, grant No. 14-25-00136).

\section{References}

1. Bliss TV, Collingridge GL (1993) A synaptic model of memory: long-term potentiation in the hippocampus. Nature 361: 31-39. [Crossref]

2. Stuchlik A (2014) Dynamic learning and memory, synaptic plasticity and neurogenesis: an update. Front Behav Neurosci 8: 106. [Crossref]

3. Fa M, Xia L, Anunu R, Kehat O, Kriebel M, et al. (2014) Stress modulation of hippocampal activity--spotlight on the dentate gyrus. Neurobiol Learn Mem 112: 5360. [Crossref]

4. Leuner B, Shors TJ (2013) Stress, anxiety, and dendritic spines: what are the connections? Neuroscience 251: 108-119. [Crossref]

5. Schmidt HD, Duman RS (2007) The role of neurotrophic factors in adult hippocampal neurogenesis, antidepressant treatments and animal models of depressive-like behavior. Behav Pharmacol 18: 391-418. [Crossref]

6. Hanson ND, Owens MJ, Nemeroff CB (2011) Depression, antidepressants, and neurogenesis: a critical reappraisal. Neuropsychopharmacology 36: 2589-2602. [Crossref]

7. Lopez-Rojas J, Kreutz MR (2016) Mature granule cells of the dentate gyrus--Passive bystanders or principal performers in hippocampal function? Neurosci Biobehav Rev 64: 167-174. [Crossref]

8. Aniol VA, Gulyaeva NV (2015) Changes in adult neurogenesis in the hippocampus during depressive disorders in humans. Neurochem $J$ 9: 8-12.

9. Schinder AF, Gage FH (2004) A hypothesis about the role of adult neurogenesis in hippocampal function. Physiology (Bethesda) 19: 253-261. [Crossref]

10. Perera TD, Park S, Nemirovskaya Y (2008) Cognitive role of neurogenesis in depression and antidepressant treatment. Neuroscientist 14: 326-338. [Crossref]

11. Brites D, Fernandes A (2015) Neuroinflammation and Depression: Microglia Activation, Extracellular Microvesicles and microRNA Dysregulation. Front Cell Neurosci 9: 476. [Crossref]
12. Kielian T (2016) Multifaceted roles of neuroinflammation: the need to consider both sides of the coin. J Neurochem 136 Suppl 1: 5-9. [Crossref]

13. Crupi R, Cambiaghi M, Spatz L, Hen R, Thorn M, et al. (2010) Reduced adult neurogenesis and altered emotional behaviors in autoimmune-prone B-cell activating factor transgenic mice. Biol Psychiatry 67: 558-566. [Crossref]

14. Tanti A, Belzung C (2013) Neurogenesis along the septo-temporal axis of the hippocampus: are depression and the action of antidepressants region-specific? Neuroscience 252: 234-252. [Crossref]

15. Gulyaeva NV (2014) Effects of Stress Factors on the Functioning of the Adult Hippocampus: Molecular-Cellular Mechanisms and the Dorsoventral Gradient. Neuroscience and Behavioral Physiology 44: 973-86.

16. Gulyaeva NV (2015) Ventral hippocampus, stress and phychopathology: Translational implications. Neurochem J 9: 85-94.

17. Dine J, Ionescu IA, Avrabos C, Yen YC, Holsboer F, et al. (2015) Intranasally applied neuropeptide $\mathrm{S}$ shifts a high-anxiety electrophysiological endophenotype in the ventral hippocampus towards a "normal"-anxiety one. PLoS One 10: e0120272. [Crossref]

18. Kudryashova PV, Gulyaeva NV (2016) Unpredictable stress»: ambiguous stressreactivity of long-term plasticity. Zh Vyssh Nerv Deiat Im I P Pavlova 66: in press

19. Kamal A, Ramakers GM, Altinbilek B, Kas MJ (2014) Social isolation stress reduces hippocampal long-term potentiation: effect of animal strain and involvement of glucocorticoid receptors. Neuroscience 256: 262-270. [Crossref]

20. Takeda A (2012) Zinc signaling in the hippocampus and its relation to pathogenesis of depression. J Trace Elem Med Biol 26: 80-84. [Crossref]

21. Lanfumey L, Mongeau R, Cohen-Salmon C, Hamon M (2008) Corticosteroid-serotonin interactions in the neurobiological mechanisms of stress-related disorders. Neurosci Biobehav Rev 32: 1174-1184. [Crossref]

22. Matsumoto M, Togashi H, Ohashi S, Tachibana K, Yamaguchi T, et al. (2004) Serotonergic modulation of psychological stress-induced alteration in synaptic plasticity in the rat hippocampal CA1 field. Brain Res 1022: 221-225. [Crossref]

23. Grigoryan GA, Gulyaeva NV (2015) [Animal Models of Depression: Behavior as the Basis for Methodology, Assessment Criteria and Classifications]. Zh Vyssh Nerv Deiat Im I P Pavlova 65: 643-660. [Crossref]

24. Adamec RE, Burton P, Shallow T, Budgell J (1999) NMDA receptors mediate lasting increases in anxiety-like behavior produced by the stress of predator exposure-implications for anxiety associated with posttraumatic stress disorder. Physiol Behav 65: 723-737. [Crossref]

25. Marsden WN (2011) Stressor-induced NMDAR dysfunction as a unifying hypothesis for the aetiology, pathogenesis and comorbidity of clinical depression. Med Hypotheses 77: 508-528. [Crossref]

26. Kudryashova IV (2015) Neurodegenerative changes in depression: Excitotoxicity or a deficit of trophic factors? Neurochem $J$ 9: 1-7

27. Tse YC, Bagot RC, Hutter JA, Wong AS, Wong TP (2011) Modulation of synaptic plasticity by stress hormone associates with plastic alteration of synaptic NMDA receptor in the adult hippocampus. PLoS One 6: e27215. [Crossref]

28. Stepanichev M, Dygalo NN, Grigoryan G, Shishkina GT, Gulyaeva N (2014) Rodent models of depression: neurotrophic and neuroinflammatory biomarkers. Biomed Res Int 14: 932757.

29. Grigoryan GA, Gulyaeva NV (2015) [Stress reactivity and stress-resilience in the pathogenesis of depressive disorders: involvement of epigenetic mechanisms]. $\mathrm{Zh}$ Vyssh Nerv Deiat Im I P Pavlova 65: 19-32. [Crossref]

30. Inoue S, Shikanai H, Matsumoto M, Hiraide S, Saito Y, et al. (2014) Metaplastic regulation of the median raphe nucleus via serotonin 5-HT1A receptor on hippocampal synaptic plasticity is associated with gender-specific emotional expression in rats. $J$ Pharmacol Sci 124: 394-407. [Crossref]

31. Li W, Liu L, Liu YY, Luo J, Lin JY, et al. (2012) Effects of electroconvulsive stimulation on long-term potentiation and synaptophysin in the hippocampus of rats with depressive behavior. JECT 28: 111-117. [Crossref]

32. Qiao H, An SC, Ren W, Ma XM (2014) Progressive alterations of hippocampal CA3CA1 synapses in an animal model of depression. Behav Brain Res 275: 191-200. [Crossref]

33. Zhang Z, Wang W, Zhong P, Liu SJ, Long JZ, et al. (2015) Blockade of 2-arachidonoylglycerol hydrolysis produces antidepressant-like effects and enhances adult hippocampal neurogenesis and synaptic plasticity. Hippocampus 25: 16-26. [Crossref] 
34. Liu M, Li J, Dai P, Zhao F, Zheng G, et al. (2015) Microglia activation regulates GluR1 phosphorylation in chronic unpredictable stress-induced cognitive dysfunction. Stress 18: 96-106. [Crossref]

35. Segev A, Rubin AS, Abush H, Richter-Levin G, Akirav I (2014) Cannabinoid receptor activation prevents the effects of chronic mild stress on emotional learning and LTP in a rat model of depression. Neuropsychopharmacology 39: 919-33. [Crossref]

36. Reich CG, Mihalik GR, Iskander AN, Seckler JC, Weiss MS (2013) Adolescent chronic mild stress alters hippocampal CB1 receptor-mediated excitatory neurotransmission and plasticity. Neuroscience 253: 444-454. [Crossref]

37. Tishkina A, Stepanichev M, Kudryashova I, Freiman S, Onufriev M, et al. (2016) Neonatal proinflammatory challenge in male Wistar rats: Effects on behavior, synaptic plasticity, and adrenocortical stress response. Behav Brain Res 304: 1-10. [Crossref]

38. Piskunov A, Stepanichev M, Tishkina A, Novikova M, Levshina I, et al. (2016) Chronic combined stress induces selective and long-lasting inflammatory response evoked by changes in corticosterone accumulation and signaling in rat hippocampus. Metab Brain Dis 31: 445-454. [Crossref]

39. Sousa VC, Vital J, Costenla AR, Batalha VL, Sebastião AM, et al. (2014) Maternal separation impairs long term-potentiation in CA1-CA3 synapses and hippocampaldependent memory in old rats. Neurobiol Aging 35: 1680-1685. [Crossref]

40. Zhang X, Wang B, Jin J, An S, Zeng Q, et al. (2014) Early deprivation reduced anxiety and enhanced memory in adult male rats. Brain Res Bull 108: 44-50. [Crossref]

41. Weinstock M (2007) Gender differences in the effects of prenatal stress on brain development and behaviour. Neurochem Res 32: 1730-1740. [Crossref]

42. Barzegar M, Sajjadi FS, Talaei SA, Hamidi G, Salami M (2015) Prenatal exposure to noise stress: anxiety, impaired spatial memory, and deteriorated hippocampal plasticity in postnatal life. Hippocampus 25: 187-196. [Crossref]

43. Yeh CM, Huang CC, Hsu KS (2012) Prenatal stress alters hippocampal synaptic plasticity in young rat offspring through preventing the proteolytic conversion of probrain-derived neurotrophic factor (BDNF) to mature BDNF. J Physiol 590: 991-1010. [Crossref]

44. Buwalda B, Kole MH, Veenema AH, Huininga M, de Boer SF, et al. (2005) Long-term effects of social stress on brain and behavior: a focus on hippocampal functioning. Neurosci Biobehav Rev 29: 83-97. [Crossref]

45. Collins DR (2009) Differential modulation of hippocampal plasticity in a non-noxious conflict model for anxiety. Neuroscience 162: 863-869. [Crossref]
46. Ardi Z, Ritov G, Lucas M, Richter-Levin G (2014) The effects of a reminder of underwater trauma on behaviour and memory-related mechanisms in the rat dentate gyrus. Int J Neuropsychopharmacol 17: 571-580. [Crossref]

47. Quan MN, Zhang N, Wang YY, Zhang T, Yang Z (2011) Possible antidepressant effects and mechanisms of memantine in behaviors and synaptic plasticity of a depression rat model. Neuroscience 182: 88-97. [Crossref]

48. Goldwater DS, Pavlides C, Hunter RG, Bloss EB, Hof PR, et al. (2009) Structural and functional alterations to rat medial prefrontal cortex following chronic restraint stress and recovery. Neuroscience 164: 798-808. [Crossref]

49. Judo C, Matsumoto M, Yamazaki D, Hiraide S, Yanagawa Y, et al. (2010) Early stress exposure impairs synaptic potentiation in the rat medial prefrontal cortex underlying contextual fear extinction. Neuroscience 169: 1705-1714. [Crossref]

50. Ito H, Nagano M, Suzuki H, Murakoshi T (2010) Chronic stress enhances synaptic plasticity due to disinhibition in the anterior cingulate cortex and induces hyperlocomotion in mice. Neuropharmacology 58: 746-757. [Crossref]

51. Adamec RE (2001) Does long term potentiation in periacqueductal gray (PAG) mediate lasting changes in rodent anxiety-like behavior (ALB) produced by predator stress?Effects of low frequency stimulation (LFS) of PAG on place preference and changes in ALB produced by predator stress. Behav Brain Res 120: 111-135. [Crossref]

52. Pollandt S, Liu J, Orozco-Cabal L, Grigoriadis DE, Vale WW, et al. (2006) Cocaine withdrawal enhances long-term potentiation induced by corticotropin-releasing factor at central amygdala glutamatergic synapses via CRF, NMDA receptors and PKA. Eur J Neurosci 24: 1733-1743. [Crossref]

53. Gee CE, Peterlik D, Neuhäuser C, Bouhelal R, Kaupmann K, et al. (2014) Blocking metabotropic glutamate receptor subtype 7 (mGlu7) via the Venus flytrap domain (VFTD) inhibits amygdala plasticity, stress, and anxiety-related behavior. $J$ Biol Chem 289: 10975-10987. [Crossref]

54. Paul S, Olausson P, Venkitaramani DV, Ruchkina I, Moran TD, et al. (2007) The striatal-enriched protein tyrosine phosphatase gates long-term potentiation and fear memory in the lateral amygdala. Biol Psychiatry 61: 1049-1061. [Crossref]

55. Abush H, Akirav I (2013) Cannabinoids ameliorate impairments induced by chronic stress to synaptic plasticity and short-term memory. Neuropsychopharmacology 38 : 1521-1534. [Crossref]

56. Conrad KL, Louderback KM, Gessner CP, Winder DG (2011) Stress-induced alterations in anxiety-like behavior and adaptations in plasticity in the bed nucleus of the stria terminalis. Physiol Behav 104: 248-256. [Crossref]

Copyright: $(02016$ Gulyaeva NV. This is an open-access article distributed under the terms of the Creative Commons Attribution License, which permits unrestricted use, distribution, and reproduction in any medium, provided the original author and source are credited. 